\title{
Study on Fusion Image Based on Discrete Orthonormal
}

\section{S-transform Technology}

\author{
Tong Shu ${ }^{1, a, *}$, Bao-Hai Yang ${ }^{2, b}$, Ming Hu ${ }^{1, c}$ \\ ${ }^{1}$ Department of Electronic Engineering, NanChang Institute of Science ,330108,China \\ ${ }^{2}$ College of Electronic Engineering, JiuJiang university , 332005,China \\ astyxmshy@qq.com, ${ }^{\mathrm{b}} 23029772$ @qq.com, ${ }^{\mathrm{c}} 7752379 @ q q . c o m$ \\ *The Corresponding author
}

Keywords: 2D-discrete orthonormal S-transform; image fusion; multiple spatial-scale

\begin{abstract}
A new image fusion technique based on 2D discrete orthonormal S-Transform (known as the 2D-DOST) is introduced in this paper: First, the source images are decomposed by 2D-DOST to obtain the low-frequency sub-band coefficients and the high-frequency sub-band coefficients; second, according to the characteristics of the low frequency sub-band coefficients and the high frequency sub-band coefficients, the principles of choosing high frequency and low frequency coefficients are discussed respectively by taking the average fusion coefficient and the maximum phase angle fusion criterion; Finally, the output image can be obtained through inverse 2D-DOST transform. This algorithm is tested by fusing classical source images. The experiment shows that the fused image can preserve the details of each input images.
\end{abstract}

\section{Introduction}

Image fusion is the process of combining relevant information from two or more complementary and redundant source images into a single image. The resulting image will be more informative than any of the input images. At present, the method of image information extraction based on time-frequency transform is the mainstream algorithm. Time-frequency transform ${ }^{[1-3]}$ which includes Fourier transform, discrete orthogonal wavelet transforms, wavelet packet transform and multi-scale geometric wavelet transform, has good performance in sparse representation of images, but there are still some defects such as spatial frequency deletion and phase information missing. In the process of image fusion, the lossless transform and representation of the source images' information is the key technology.

2D-DOST ${ }^{[4]}$ inherits two-dimensional $S$ transform's time-frequency characteristics and its absolute reference phase information, it has the same progressive resolution as the wavelet transform and has the same spectral characteristics as the Flourier transform. 
per, a novel image fusion approach has been presented which utilizes the 2D-DOST to extract the geometric structure, spatial frequency and directional information of the source image. The rest of the paper is as follow: section 2 and 3 briefly presents 2D-ST and 2D-DOST; section 4 describes the proposed algorithm based on 2D-DOST; section 5 discusses the experiments and section 6 draws the conclusion.

\section{D Stockwell Transform (2D-ST)}

2D Stockwell Transform (2D-ST) is a relatively new algorithm of linear time-frequency transform, in which the frequency resolution varies with the frequency of the signal. For a $2 \mathrm{D}$ image $\mathrm{f}(\mathrm{x}, \mathrm{y}), 2 \mathrm{D}-\mathrm{ST}$ is defined as follows:

$S\left(\mathrm{u}, \mathrm{v}, \mathrm{f}_{u}, \mathrm{f}_{\nu}\right)=\int_{-\infty}^{\infty} \int_{-\infty}^{\infty} f(\mathrm{x}, \mathrm{y}) \frac{\left|f_{u}\right|\left|\mathrm{f}_{v}\right|}{2 \pi} \cdot e^{\left[\frac{(u-x)^{2} f_{u}^{2}+(v-y)^{2} f_{v}^{2}}{2}\right]} \cdot e^{\left[-i 2 p\left(f_{u} x+f_{v} y\right)\right]} d x d y$

Where $u$ and $v$ are the shift parameters controlling the center position of Gaussian windows on the $x$-axis and $y$-axis, respectively; while $f_{u}$ and $f_{v}\left(f_{u} \neq 0 ; f_{v} \neq 0\right)$ are frequencies related to the scale parameters in two directions, which respectively control the frequency expansion space of the window in $x$ and $y$ directions.

Its inverse transformation is defined as follow:

$$
F\left(f_{u}, f_{v}\right)=\int_{-\infty}^{\infty} \int_{-\infty}^{\infty} S\left(u, v, f_{u}, f_{v}\right) d u d v
$$

That is, summing up along the $\mathrm{X}$ axis and the $\mathrm{Y}$ axis, and then obtaining the source image by Fourier inverse transformation.

\section{D Discrete Orthonormal S-Transform (2D-DOST)}

For $2 \mathrm{D}-\mathrm{ST}$, the major drawback is that its time-frequency information is redundant and the calculation is complex. To improve computing efficiency, 2D-DOST is proposed: it divides the time frequency domain into $\mathrm{N}$ regions (corresponding $\mathrm{N} \mathrm{x} \mathrm{N}$ images), and each region is represented by a coefficient.

The 2D-DOST of an $\mathrm{N} \times \mathrm{N}$ image $\mathrm{f}(\mathrm{x}, \mathrm{y})$ is defined as:

$$
S\left(\mathrm{u}, \mathrm{v}, \mathrm{v}_{x}, \mathrm{v}_{y}\right)=\frac{1}{\sqrt{2^{p_{x}-p_{y}-2}}} \cdot \sum_{m=-2^{p_{x}-2}}^{2^{\rho_{x}-2}-1} \sum_{n=-2^{p_{y}-2}}^{2^{p_{y}-2}} F\left(\mathrm{~m}+\mathrm{v}_{x}, \mathrm{n}+\mathrm{v}_{y}\right) \cdot e^{2 \pi i\left(\frac{m u}{2^{p_{x}-1}}+\frac{n V}{2^{p_{y}-1}}\right)}
$$

Where:

$F(\mathrm{~m}, \mathrm{n})$ :the Fourier transform of the image $f(\mathrm{x}, \mathrm{y})$; 


$$
\text { a }
$$


All variations of $2 \mathrm{D}$-DOST look very similar when applied to an image. There are slight differences between the partitioning and actual coefficient values, but the large-scale features are the same. The 2D-DOST of an image is shown in figure 2. The zero-frequency component segments at the center of the figure, with frequency increasing to the edge.

A miniature copy of the image is seen in each sub-band. This is due to the stronger correlation of the basic functions to edges, particularly for higher frequencies. A similar effect is seen in quad-tree representations of wavelet transforms. The image in bands with negative frequencies is reflected, because the order of coefficients is reversed to maintain conjugate-symmetry.

\section{Image fusion algorithm Using 2D-DOST}

The 2D-DOST transform can effectively preserve the spatial information and frequency information of image, which is beneficial to the information fusion of the source image.

The proposed average-max-based image fusion method using 2D-DOST (Figure 3) is as follows:

Step1: according to Eq. (3), transform the source image A and B to get its 2D-DOST coefficients $S_{A}\left(u, v, v_{x}, v_{y}\right), S_{B}\left(u, v, V_{x}, v_{y}\right)$; assuming the fused image is $\mathrm{F}$, the corresponding 2D-DOST coefficients are $S_{F}\left(u, v, V_{x}, V_{y}\right)$.

$S_{Z}^{L}\left(u, v, V_{x}, v_{y}\right)(\mathrm{Z}=\mathrm{A}, \mathrm{B}, \mathrm{F})$ is the low frequency sub-band 2D-DOST coefficient of image $\mathrm{Z}$ and $S_{Z}^{H}\left(u, v, v_{x}, v_{y}\right)(\mathrm{Z}=\mathrm{A}, \mathrm{B}, \mathrm{F})$ is the high frequency sub-band 2D-DOST coefficient of image $\mathrm{Z}$.

Step2: Figure out $T_{A}^{H}\left(u, v, v_{x}, v_{y}\right), T_{B}^{H}\left(u, v, v_{x}, v_{y}\right)$ the high frequency sub-band 2D-DOST phase angle of $S_{A}^{H}\left(u, v, v_{x}, v_{y}\right), S_{B}^{H}\left(u, v, v_{x}, v_{y}\right)$.

Step3: gets the fusion coefficients $S_{\mathrm{F}}^{\mathrm{L}}\left(u, v, v_{x}, v_{y}\right)$ and $S_{\mathrm{F}}^{H}\left(u, v, v_{x}, v_{y}\right)$ according to the following fusion rule:

For low frequency, the fusion coefficient is the average value of source images' corresponding coefficients.

$S_{\mathrm{F}}^{\mathrm{L}}\left(u, v, v_{x}, v_{y}\right)=\frac{1}{2} \times\left[S_{\mathrm{A}}^{\mathrm{L}}\left(u, v, v_{x}, v_{y}\right)+S_{\mathrm{B}}^{\mathrm{L}}\left(u, v, v_{x}, v_{y}\right)\right] \quad$ For high frequency,

If $T_{A}^{H}\left(u, v, V_{x}, V_{y}\right) \geq T_{B}^{H}\left(u, v, v_{x}, v_{y}\right)$, then $S_{F}^{H}\left(u, v, v_{x}, v_{y}\right)=S_{A}^{H}\left(u, v, V_{x}, v_{y}\right)$;

If $T_{A}^{H}\left(u, v, v_{x}, v_{y}\right)<T_{B}^{H}\left(u, v, v_{x}, v_{y}\right)$, then $S_{F}^{H}\left(u, v, v_{x}, v_{y}\right)=S_{B}^{H}\left(u, v, v_{x}, v_{y}\right)$. 
Step4: according to Eq. (4), inverse transform $S_{F}^{L}\left(u, v, v_{x}, v_{y}\right), S_{F}^{H}\left(u, v, v_{x}, v_{y}\right)$ and obtain the fused image $F$.

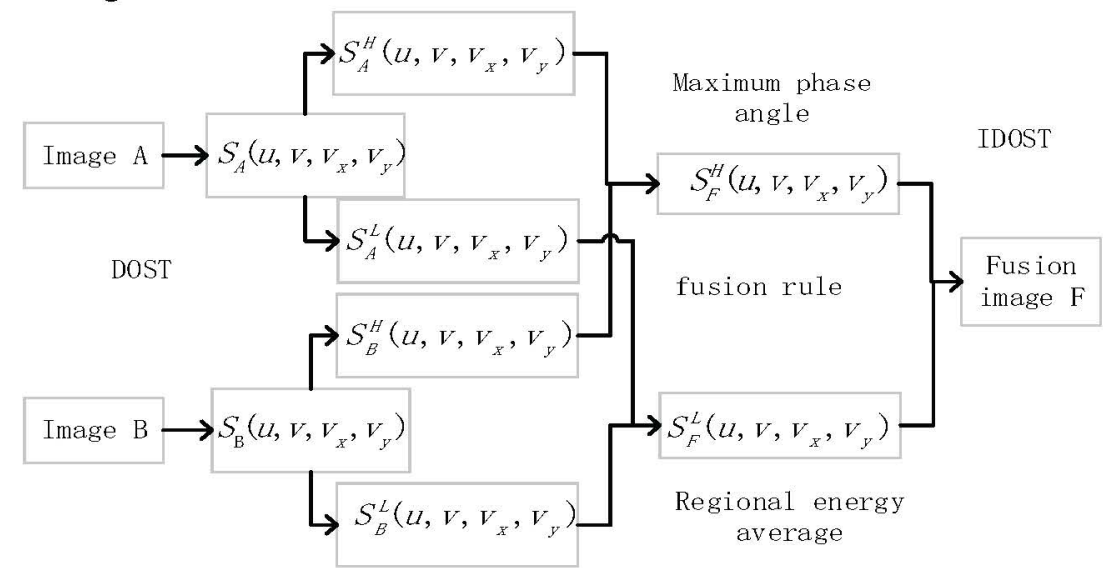

Fig.3, The flow diagram of 2D-DOST

\section{Simulation Experiments}

In experiments, the performance of proposed 2D-DOST algorithm was evaluated in fusion images modes, which gives a spatial-frequency decomposition with no redundancy. The fast Fourier transform (FFT) and the discrete orthogonal wavelet transform (DWT) were used for compare. All of the three transform algorithms adopted the image fusion strategy proposed in Figure 3,e and three parameters: $Q^{A B / F[10]}$, $M I^{A B / F}[11]$ and BSSIM ${ }^{[12]}$ were used to evaluate the quality of fused images. Among them: $Q^{A B / F}$ reflects the edge information of an image, and its larger value indicates the better edge features of the fusion image; $M I^{A B / F}$ is an important index to measure how much information is fused together, the larger the value is, the more information is merged; BSSIM reflects the structural similarity between the fused image and the source image, and the larger value indicates the better similarity between them.

We applied these three methods to fuse three different test source images (shown in Figure $4,5,6)$. The test source images are all $256 \times 256$ pixels in size. Therefore, the frequency order $\left(p_{x}, p_{y}\right)$ can have the values in range $0,1 \ldots, 7$.

Figure 4 (a),(b) are the source images of CT and MR in the same brain regions; Figure 5(a), (b) are the fuzzy clock source images; Figure 6 (a), (b) are source images in infrared light and natural light; Figure 4(c), 5(c), 6(c) are the 2D-DOST fusion image; Figure 4(d), 5(d), 6(d) are the DWT fusion image; Figure 4(e), 5(e), 6(e) are the FFT fusion image.

In figure 4(c), the fused image clearly displayed both the information of bone tissue (CT) and that of soft tissue (MR) at the same time. The edges and textures are clear and the contrast ratio is high. In figure 5(c), the 2D-DOST algorithm improved the entropy of 
the fused image, kept the features of source images. The alarm clocks is clearer. In figure 6(c), the fused image is distinctly segmented into multi-regions, the image edge is legible, and the fusion result is satisfactory.

The experimental results show that the proposed algorithm can fuse the multi-source images effectively, and can effectively extract the geometric structure, spatial frequency and directional information of the source images.

Table 1 recorded the $Q^{A B / F} 、 M^{A B / F}$ and BSSIM of fused images for the experiment. In all cases, the 2D-DOST method yields a substantially higher than the FFT and DWT methods.

Easy to see, the $Q^{A B / F}$ index value of 2D-DOST is equal to that of FFT but larger than that of DWT, which indicates that the 2D-DOST and FFT fusion images have better edge features than DWT fusion image. The 2D-DOST is obtained by combining FFT with traditional ST, and this is the reason why 2D-DOST and FFT has the same $Q^{A B / F}$ value. In table 1 , the numerical value of $Q^{A B / F}$ indirectly proved that $2 \mathrm{D}$-DOST has the same frequency characteristics as FFT.

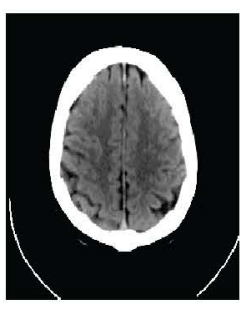

(a)

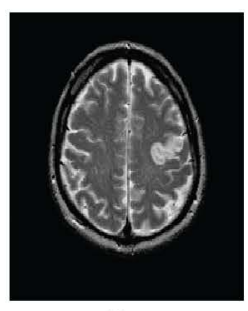

(b)

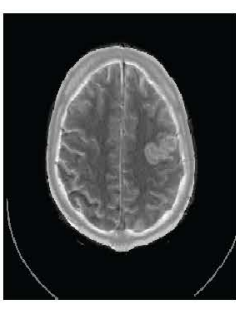

(c)

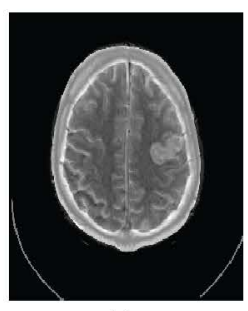

(d)

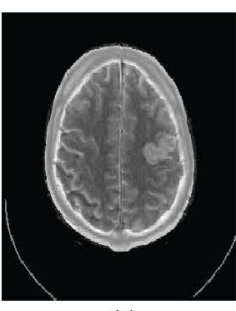

(e)

Fig.4, The fusion results of Brain CT and MR images by different algorithms

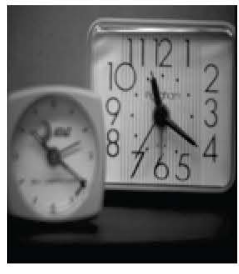

(a)

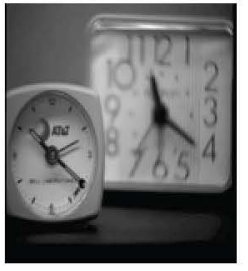

(b)

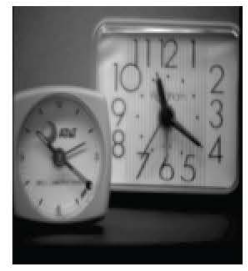

(c)

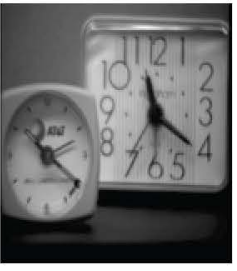

(d)

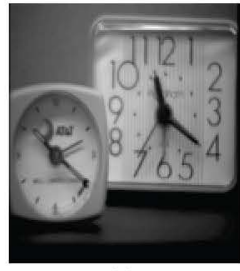

(e)

Fig.5, The fusion results of clock images by different algorithms

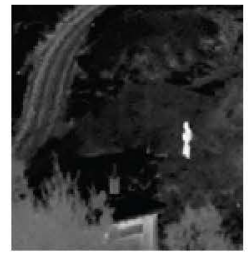

(a)

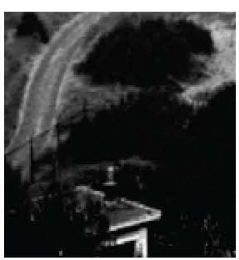

(b)

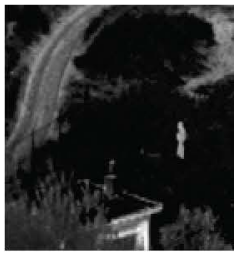

(c)

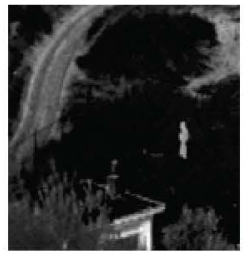

(d)

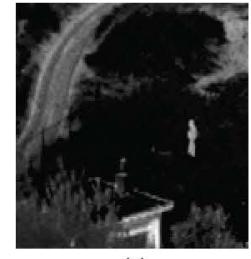

(e)

Fig.6, The fusion results of Infrared and natural images by different algorithms

In figure 4 and figure 6: the BSSIM, $\mathrm{MI}^{A B / F}$ index values based on 2D-DOST are larger than the corresponding values of the other two kinds of algorithm, shows that 
2D-DOST fused more information than the other two algorithms, and the fusion images are more similar to the original images.

Tab.1 Objective indexes of three algorithms

\begin{tabular}{ccccc}
\hline $\begin{array}{c}\text { fusion } \\
\text { image }\end{array}$ & $\begin{array}{c}\text { transform } \\
\text { method }\end{array}$ & $Q^{A B / F}$ & MI $^{A B / F}$ & BSSIM \\
\hline Figure & 2D-DOST & $\mathbf{0 . 7 2 7 7}$ & $\mathbf{4 . 6 6 7 0}$ & $\mathbf{0 . 4 0 4 9}$ \\
4 & FFT & 0.7277 & 4.6659 & 0.3853 \\
& DWT & 0.7261 & 4.6506 & 0.4042 \\
Figure & 2D-DOST & $\mathbf{0 . 9 3 3 6}$ & 7.0026 & 0.5843 \\
5 & FFT & 0.9336 & 7.0354 & 0.5860 \\
& DWT & 0.9333 & $\mathbf{7 . 1 8 8}$ & $\mathbf{0 . 5 9 4 3}$ \\
Figure & 2D-DOST & $\mathbf{0 . 7 5 1 5}$ & $\mathbf{2 . 6 6 6}$ & $\mathbf{0 . 3 4 3 1}$ \\
6 & FFT & 0.7515 & 2.6594 & 0.3413 \\
& DWT & 0.7510 & 2.6055 & 0.3421 \\
\hline
\end{tabular}

In Figure 5, the 2D-DOST parameter does not perform well. Comparing Fig. 5 with Fig. 4 and Fig.6, we found that the black and white mutation is more prominent in Fig.5. 2D-DOST inherits the frequency characteristic of FFT and its corresponding value of phase angle is large. In this case, if we still take the above fusion criterion, it will extract more sharp change informations than flat change informations and thus resulting in the loss of part of the image fusion information. So, when an image with different gray gradient is fused by maximum phase method, the bandwidth of high frequency sub-band should be selected reasonably.

\section{Conclusions}

In image fusion, the fused image is required to reflect all the information contained in source images as accurately as possible. 2D-DOST simultaneously has multi-scale resolution property of DWT and phase information of FFT. For the first time, this paper proposed the application of 2D-DOST algorithm in image fusion field. The experimental results show that the fusion image details and edge information based on the proposed algorithm is clear, and the output image is similar to the input images; both the parameters and visual effects show that the applicability of 2D-DOST in the field of image fusion. This paper is a preliminary study of the application of 2D-DOST in image fusion, yet the image fusion strategy needs to be further researched.

\section{Acknowledgment}

The work has been supported by the natural science foundation of Jiangxi Province (Grant No. 20151BAB207013), research foundation of health department of Jiangxi province China (Grant No.20175561), science foundation of jiangxi provincial department of education (Grant No. GJJ161067) ,science foundation of jiujiang university (Grant No.2016KJ001,2016KJ003). 


\section{Reference}

[1] Nikolov S, Hill P, Bull D, et al. Wavelets for Image Fusion[M]// Wavelets in Signal and Image Analysis. Springer Netherlands, 2001:213-241.

[2] Chipman L J, Orr T M, Graham L N. Wavelets and image fusion[C]// International Conference on Image Processing. IEEE Computer Society, 1995:3248.

[3] NIKOLOV S, HILL P, BULL D, et al. Wavelets for Image Fusion [M]. Springer Netherlands, 2001.

[4] STOCKWELL R G. A basis for efficient representation of the S-transform [J]. 2007, 17(1): 371-93.

[5] CHORAŚ R S. Time-Frequency Analysis of Image Based on Stockwell Transform [M]. Springer International Publishing, 2014.

[6] SUN F R, BABYN P, LUAN Y H, et al. Image Denoising Using Discrete Orthonormal S-Transform [J]. 2014, 250(435-42).

[7] HUANG H, SUN F, BABYN P, et al. Medical Image Denoising and Compressing Using Discrete Orthonormal S-transform [J]. 2015,

[8] SAEDI S, CHARKARI N M. Characterization of Palmprint Using Discrete Orthonormal S-Transform; proceedings of the International Conference on Hand-Based Biometrics, F, 2011 [C].2011

[9] DRABYCZ S, STOCKWELL R G, MITCHELL J R. Image texture characterization using the discrete orthonormal S-transform [J]. Journal of Digital Imaging, 2009, 22(6): 696.

[10]QU G, ZHANG D, YAN P. Information measure for performance of image fusion [J]. Electronics Letters, 2002, 38(7): 313-5.

[11]XYDEAS C S, PETROVIC V. Objective image fusion performance measure [J]. Military Technical Courier, 2000, 36(4): 308-9.

[12] WANG Z, BOVIK A C, SHEIKH H R, et al. Image quality assessment: from error visibility to structural similarity [J]. IEEE Transactions on Image Processing, 2004, 13(4): $600-12$. 